\title{
Continuous Femoral Nerve Block Provides Superior Analgesia Compared With Continuous Intra-articular and Wound Infusion After Anterior Cruciate Ligament Reconstruction
}

\author{
Mario Dauri, MD, * Eleonora Fabbi, MD, * Pierpaolo Mariani, MD, † Skerdilajd Faria, MD, * \\ Roberta Carpenedo, MD, * Tatiana Sidiropoulou, MD, $\neq$ Filadelfo Coniglione, MD, * \\ Maria B. Silvi, MD, * and Alessandro F. Sabato, MD*
}

\begin{abstract}
Background and Objectives: This prospective, randomized, clinical trial compared pain intensity and analgesic drug consumption after anterior cruciate ligament (ACL) reconstruction with patellar tendon under femoral-sciatic nerve block anesthesia in patients who received either a continuous femoral nerve block (CFNB) or continuous local anesthetic wound and intra-articular infusions.
\end{abstract}

Methods: Fifty patients were randomized to CFNB $(n=25)$ or an ON$\mathrm{Q}$ device (I-Flow Corp, Lake Forest, Calif) $(\mathrm{n}=25)$. All patients received sciatic nerve block $(25 \mathrm{~mL}$ of ropivacaine $7.5 \mathrm{mg} / \mathrm{mL}$ and clonidine $30 \mu \mathrm{g})$. The first group received a CFNB $(2 \mathrm{mg} / \mathrm{mL}$ of ropivacaine at $7 \mathrm{~mL} / \mathrm{hr}$ ), and the second group received a single-shot femoral nerve block (both using $25 \mathrm{~mL}$ of ropivacaine $7.5 \mathrm{mg} / \mathrm{mL}$ and clonidine $30 \mu \mathrm{g}$ ). At the end of the intervention, an ON-Q device was positioned on the ON-Q patients to continuously infuse the patellar tendon wound and intra-articular cavity with ropivacaine $2 \mathrm{mg} / \mathrm{mL}$ at $2 \mathrm{~mL} / \mathrm{hr}$ for each catheter. Data regarding demographic, hemodynamic, pain scores, adverse effects, and need for supplemental analgesia were registered in a 36-hr follow-up period.

Results: The CFNB group reported lower visual analog scale values than the ON-Q group: at rest at $12 \mathrm{hrs}(2.4$ [SD, 2.2] vs 5.4 [SD, 3.1]; $P<0.001)$ and on movement at $12(3.1[\mathrm{SD}, 2.5]$ vs 6.3 [SD, 2.9]; $P<0.001)$ and $24 \mathrm{hrs}(2.7[\mathrm{SD}, 1.9]$ vs $4.6[\mathrm{SD}, 2.6] ; P=0.01)$ after surgery. The number of morphine and ketorolac boluses was lower in the CNFB group (morphine: 3.2 [SD, 2.2] vs 6.2 [SD, 2.5]; $P<0.001$; ketorolac: 1.1 [SD, 1.0] vs 2.4 [SD, 0.9]; $P<0.001$ ).

Conclusion: Continuous femoral nerve block provides better analgesia than the continuous patellar tendon wound and intra-articular infusions after anterior cruciate ligament reconstruction with patellar tendon.

(Reg Anesth Pain Med 2009;34: 95-99)

A rthroscopic anterior cruciate ligament (ACL) reconstruction of the knee is a painful procedure requiring intensive

From the *Department of Anesthesia and Intensive Care Medicine, University Hospital of "Tor Vergata"; †Department of Sport Traumatology, University Institute of Motor Sciences, Rome, Italy; and $\$ 2$ nd Department of Anaesthesiology, University of Athens, "Attikon" Hospital, Athens, Greece. Accepted for publication January 23, 2007.

Address correspondence to: Mario Dauri, MD, Via Di S. Eufemia, 11, 00187 Rome, Italy (e-mail: mario.dauri@fastwebnet.it).

The work should be attributed to the Department of Anesthesia and Intensive Care Medicine, University Hospital of "Tor Vergata", Rome, Italy.

This study was originally accepted for publication on January $27,2007$. Because of a processing error on the part of the journal, publication was unintentionally delayed until now. The editor-in-chief and the managing editor express their sincere apologies to the authors for this unfortunate error.

Copyright (C) 2009 by American Society of Regional Anesthesia and Pain Medicine

ISSN: $1098-7339$

DOI: 10.1097/AAP.0b013e31819baf98 postoperative pain management. The routine use of continuous femoral nerve block (CFNB) in patients undergoing ACL reconstruction provides substantially improved postoperative analgesia. ${ }^{1}$ A device delivering a constant flow of local anesthetic into the articular cavity has been effective for analgesia after shoulder surgery. ${ }^{2}$ Alford and Fadale ${ }^{3}$ concluded that continuous bupivacaine infusion seems to have a protective effect at maximum pain levels after ACL reconstruction. Hoenecke et $\mathrm{al}^{4}$ report that continuous bupivacaine infiltration in the donor site of the patellar tendon after ACL reconstruction results in good pain relief. Schwarz et al found no effect of a femoral nerve block (FNB) with ropivacaine $(2 \mathrm{mg} / \mathrm{mL})$ on postoperative analgesic consumption, compared with intra-articular instillation of ropivacaine in patients undergoing ACL reconstruction under general anesthesia. ${ }^{5}$ However, other authors report that, compared with intra-articular injection of local anesthetic, FNB provides better analgesia and allows significant morphinesparing effect after ACL repair. ${ }^{6}$

The ON-Q pain management system (I-Flow Corp, Lake Forest, Calif) consists of an elastomeric pump that holds a local anesthetic and delivers it automatically through 1 or 2 small catheters and provides an even, continuous distribution of local anesthetic over a wide surgical area.

To the best of our knowledge, there are no studies evaluating the effectiveness of continuous local anesthetic delivery with ON-Q pump or comparing it with CFNB in patients undergoing ACL reconstruction. Therefore, we designed a prospective, randomized, clinical trial to compare pain and analgesic drug consumption after ACL reconstruction with patellar tendon, in patients receiving either a CFNB or both continuous patellar tendon wound and intra-articular infusion of local anesthetic using an ON-Q pain management system immediately after a surgical procedure performed with regional anesthesia (femoral and sciatic nerve block).

\section{MATERIALS AND METHODS}

After institutional ethics board approval and written informed consent, 50 American Society of Anesthesiologists physical status I adult patients with body mass index (BMI) from 20 to $30 \mathrm{~kg} / \mathrm{m}^{2}$ scheduled to undergo elective arthroscopically assisted ACL reconstruction with the middle third bone-patellar tendon-bone autograft technique were prospectively enrolled in this study. Exclusion criteria were as follows: hypersensitivity or known allergy to any of the study drugs, recent opioid use or alcohol abuse, presence of any contraindication to regional anesthesia, pre-existing coagulation disorder, diabetic or femoral neuropathy, or prior surgery in the inguinal region. Patients were randomized by a computer-generated list into CFNB or ON-Q groups. All patients were premedicated with $5 \mu \mathrm{g}$ of sufentanil 5 mins before anesthesia. Both groups received a singleinjection sciatic nerve block via the subgluteal approach; the 
ON-Q group $(\mathrm{n}=25)$ then received a single-injection FNB, whereas the CFNB group $(n=25)$ received a CFNB.

\section{Sciatic Nerve Block}

After aseptic skin disinfection of the gluteal region of the affected limb, a skin wheal was raised with 1 to $2 \mathrm{~mL}$ of 20 $\mathrm{mg} / \mathrm{mL}$ lidocaine. A 120-mm, 21-gauge stimulation needle (Polymedic; Temena SARL, Bondy, France) connected to a peripheral nerve stimulator (Stimuplex; B. Braun, Melsungen, Germany) with initial current intensity of $1 \mathrm{~mA}(2 \mathrm{~Hz}, 0.1$ millisecond) was inserted perpendicular to the skin and advanced until motor responses of the posterior tibial component (plantar flexion or inversion) were elicited. The current output was progressively diminished until contractions were obtained at an intensity of 0.3 to $0.4 \mathrm{~mA}$. Twenty-five milliliters of ropivacaine $7.5 \mathrm{mg} / \mathrm{mL}$ and clonidine $30 \mu \mathrm{g}$ were then injected through the needle.

\section{Continuous Femoral Catheter Placement Techniques}

After aseptic skin disinfection and sterile draping of the inguinal region of the affected limb, a skin wheal was raised with 1 to $2 \mathrm{~mL}$ of $20 \mathrm{mg} / \mathrm{mL}$ lidocaine. The initial stimulating needle insertion site was immediately below the inguinal crease, 1 to $2 \mathrm{~cm}$ lateral to the femoral artery pulsation. A 50 - $\mathrm{mm}, 18$-gauge, insulated stimulating needle (Polymedic $\mathrm{C}-50 \mathrm{~K}+$; Temena SARL) was connected to the peripheral nerve stimulator with an initial current output of $1 \mathrm{~mA}(2 \mathrm{~Hz}, 0.1$ millisecond $)$. The stimulating needle was inserted at a 45-degree angle and advanced in a cephalad direction until quadriceps femoris muscle contractions were elicited (as shown by cephalad patellar movements). The needle position was adjusted until quadriceps femoris contractions were elicited at a current of $0.4 \mathrm{~mA}$ or less. At this point, $5 \mathrm{~mL}$ of ropivacaine $7.5 \mathrm{mg} / \mathrm{mL}$ was injected; subsequently, a 20-gauge catheter was introduced through the needle and advanced for 10 to $15 \mathrm{~cm}$ beyond the needle tip, the needle was withdrawn, and the catheter was secured in place. Then, $20 \mathrm{~mL}$ of ropivacaine $7.5 \mathrm{mg} / \mathrm{mL}$ and clonidine $30 \mu \mathrm{g}$ were injected slowly through the catheter.

\section{Single-Injection Femoral Nerve Block}

This procedure was performed by the injection of $25 \mathrm{~mL}$ of ropivacaine $7.5 \mathrm{mg} / \mathrm{mL}$ and clonidine $30 \mu \mathrm{g}$ through a $30-\mathrm{mm}$, 21-gauge stimulation needle (Polymedic, Temena SARL) and was similar to the CFNB described above (without the sterile draping of the inguinal region).

\section{Continuous Local Anesthetic Infusion Technique (ON-Q Group)}

The surgeon placed sterilely the $2 \mathrm{ON}-\mathrm{Q}$ catheters at the end of the surgery. The first catheter was introduced into the articular cavity, and the second catheter was placed in the subcutaneous tissue along the patellar tendon incision site. Both catheters were first tested to be patent by the injection of $5 \mathrm{~mL}$ of ropivacaine $2 \mathrm{mg} / \mathrm{mL}$ using a syringe and then were attached to the ON-Q elastomeric infusion pump (ON-Q Pain Relief System; I-Flow Corp). The elastomeric reservoir of this infusion pump was filled with $270 \mathrm{~mL}$ of ropivacaine $2 \mathrm{mg} / \mathrm{mL}$ that was delivered continuously at a fixed rate of $2 \mathrm{~mL} / \mathrm{hr}$ through each of the 2 soaker catheters.

\section{Postoperative Analgesia}

A postoperative analgesia infusion containing ropivacaine $2 \mathrm{mg} / \mathrm{mL}$ using an infusion pump (BodyGuard 575 Pain Man- ager; CME LLC, New York, NY) at a rate of $7 \mathrm{~mL} / \mathrm{hr}$ was started as soon as patients belonging to the CFNB group were transferred to the postanesthesia care unit and continued for the next 36 hrs. Patients were also allowed patient-controlled regional analgesia (PCRA) boluses of $7 \mathrm{~mL}$ each of the local anesthetic solution, with a lockout period of $2 \mathrm{hrs}$. Patients were instructed to self-administer a PCRA bolus whenever they started to feel pain or discomfort from the surgical wound. The postoperative analgesia protocol of both groups also included supplementary analgesic treatment (rescue treatment) administered by a patient-controlled analgesia pump (BodyGuard 575 Pain Manager; CME LLC), programmed to deliver a 3-mg bolus of morphine with a 20 -min lockout time without a background morphine infusion, and 15-mg maximal dose over $2 \mathrm{hrs}$. Furthermore, additional intravenous ketorolac tromethamine (Toradol; Recordati Pharm, Milan, Italy) boluses of $30 \mathrm{mg}$ each were administered if a visual analog scale (VAS) of more than $4.0 \mathrm{~cm}$ was reported by the patient. The daily ketorolac dosage was limited to $90 \mathrm{mg}$.

No general anesthesia was performed, but sedation was carried out by continuous propofol infusion as appropriate for the anesthetic or if the patient requested it explicitly. Patients were monitored by continuing 3-lead electrocardiogram, noninvasive blood pressure, and pulse oximetry. The surgery was performed by the same surgeon, and the surgical technique was identical with intraoperative use of a thigh tourniquet inflated to $300 \mathrm{~mm} \mathrm{Hg}$ in all patients. At the end of the surgery, 2 drainages attached to $400-\mathrm{mL}$ negative pressured reservoirs (Drenofast; Iberhosèpitex SA Lliçà del Vall, Barcelona, Spain) were placed: the first into the articular cavity and the second in the patellar tendon wound. The patients were mobilized within $24 \mathrm{hrs}$ of surgery and were instructed in the use of crutches and a standard set of exercises by the physical therapy staff. All patients were discharged on the second postoperative day, after discontinuation of all analgesia regimens.

\section{Data Collection}

Demographic (age, sex, weight, height) data, surgery, and tourniquet duration were recorded. Before the anesthesia and during the postoperative period (at 2, 6, 12, 24, and $36 \mathrm{hrs}$ ), patients were monitored for pain intensity using VAS $10 \mathrm{~cm}$ long, ranging from 0 (no pain) to 10 (worst possible pain) and a 5-point Verbal Pain Scores (VS; $0=$ no pain, $1=$ mild pain, $2=$ moderate pain, $3=$ severe pain, $4=$ very severe pain) at rest and during movement of the affected limb. Hemodynamic parameters (heart rate and systolic and diastolic arterial pressure) were registered before the anesthesia, every 15 mins during the intervention, then at $2,612,24$, and $36 \mathrm{hrs}$ after surgery. Additional analgesics or sedatives such as sufentanil or propofol

TABLE 1. Patient Demographics and Tourniquet and Surgery Duration

\begin{tabular}{lccc}
\hline Characteristics & \multicolumn{1}{c}{ CFNB } & \multicolumn{1}{c}{ ON-Q } & $\boldsymbol{P}$ \\
\hline Age, y & $29(7) ; 18-41$ & $28(8) ; 18-45$ & 0.4 \\
Sex, M/F & $25 / 1$ & $26 / 0$ & 0.5 \\
BMI, $\mathrm{kg} / \mathrm{m}^{2}$ & $24(2) ; 20-28$ & $23(1.5) ; 21-26$ & 0.3 \\
Tourniquet duration, min & $56(16) ; 30-102$ & $56(6) ; 47-74$ & 0.3 \\
Surgery duration, min & $69(18) ; 50-120$ & $74(14) ; 55-115$ & 0.07 \\
\hline \multicolumn{4}{c}{ Data are reported as mean (SD); range. } \\
\multicolumn{2}{l}{ M indicates male; F, female. } \\
\hline
\end{tabular}


TABLE 2. Analgesia Monitoring During the Postsurgery Period

\begin{tabular}{|c|c|c|c|c|c|c|}
\hline \multirow[b]{2}{*}{ Follow-up } & \multicolumn{3}{|c|}{ VAS at Rest } & \multicolumn{3}{|c|}{ VAS on Movement } \\
\hline & CFNB & ON-Q & $P$ & CFNB & ON-Q & $P$ \\
\hline Presurgery & $0.1(0.3) ; 0-1$ & $0.2(0.4) ; 0-1.5$ & 0.4 & $0.4(0.3) ; 0-2$ & $0.5(0.4) ; 0-2$ & 0.5 \\
\hline Postsurgery & $0(0) ; 0-0$ & $0(0) ; 0-0$ & - & $0(0) ; 0-0$ & $0(0) ; 0-0$ & - \\
\hline $2 \mathrm{hrs}$ & $0.3(1) ; 0-2.5$ & $0.1(0.2) ; 0-0.5$ & 0.9 & $0.5(1) ; 0-3$ & $0.2(0.3) ; 0-1$ & 0.6 \\
\hline $6 \mathrm{hrs}$ & $0.6(1) ; 0-3$ & $0.5(1) ; 0-4$ & 0.5 & $1(1) ; 0-4$ & $1(1) ; 0-4$ & 0.3 \\
\hline $12 \mathrm{hrs}$ & $2(2) ; 0-7$ & $6(3) ; 0-10$ & $<0.001$ & $3(2) ; 0-8$ & $6(3) ; 0-10$ & $<0.001$ \\
\hline $24 \mathrm{hrs}$ & $2(2) ; 0-7$ & $3(2) ; 0-6$ & 0.08 & $3(2) ; 0-7$ & $4(3) ; 0-8$ & 0.03 \\
\hline $36 \mathrm{hrs}$ & $2(2) ; 0-6$ & $2(2) ; 0-5$ & 0.2 & $3(2) ; 0-6$ & $3(3) ; 0-7.6$ & 0.7 \\
\hline
\end{tabular}

Data are reported as mean $(\mathrm{SD})$; range.

administered intraoperatively based on the patient's needs were also recorded. Postoperative motor block of the operated leg was recorded using the Bromage scale as follows: $0=$ free movement of leg and foot (nil $=0 \%) ; 1=$ just able to flex knee with free movement of foot (partial $=33 \%$ ); 2 = unable to flex knee, but with free movement of foot (almost complete $=66 \%$ ); and $3=$ unable to move leg and foot (complete $=100 \%$ ). The overall boluses of ropivacaine (PCRA), morphine (patient-controlled analgesia), and ketorolac and incidence of adverse effects (nausea, vomiting, pruritus, sedation, headache, hypotension, and urinary retention) were also recorded.

\section{Sample Size and Statistical Analysis}

To calculate the sample dimension, we accepted $\alpha$ error $=$ 0.05 (type I error probability for a 2 -sided independent $t$ test) and $\beta$ error $=0.2$ (power $=80 \%$ ). Our hypothesis was to find $2.0 \mathrm{~cm}$ of difference on mean VAS after surgery between 2 groups $(\delta)$ with an $\mathrm{SD}(\sigma)$ equal to $2.5 \mathrm{~cm}$. For statistical confirmation of this hypothesis, we calculated that it would be necessary to enroll 52 patients (26 for each group). Because we had already found a highly significant difference in pain values between the groups initially, we enrolled only 50 patients. However, to observe strictly the sample size condition, we enrolled 2 other patients in a post hoc manner and assigned them randomly to one of the groups. Data in text and tables are reported as mean (SD); range. On several occasions, the variances between groups were not homogenous (we used Bartlett test for inequality of population variances); thus, we turned to the Mann-Whitney $U$ test to check differences between numeric variables. Categorical differences were tested using the Fischer exact method. All $P$ values were 2 -tailed, and $P<0.05$ was considered statistically significant. Input data and statistical analysis were performed by using Epi Info Version 3.3.2 software (Centers for Disease Control, Atlanta, Ga).

TABLE 3. Verbal Pain Scores at Rest and on Movement and the Degree of Leg Block (Bromage Scale) in the Postoperative Period

\begin{tabular}{|c|c|c|c|c|c|c|c|c|c|c|}
\hline & \multicolumn{2}{|c|}{$2 \mathrm{hrs}$} & \multicolumn{2}{|c|}{$6 \mathrm{hrs}$} & \multicolumn{2}{|c|}{$12 \mathrm{hrs}$} & \multicolumn{2}{|c|}{24 hrs } & \multicolumn{2}{|c|}{36 hrs } \\
\hline & CFNB & ON-Q & CFNB & ON-Q & CFNB & ON-Q & CFNB & ON-Q & CFNB & ON-Q \\
\hline \multicolumn{11}{|l|}{ VS* } \\
\hline No pain & $22(85)$ & $23(89)$ & $19(73)$ & $16(61)$ & $7(27)$ & $2(8)$ & $5(19)$ & $2(8)$ & $3(11)$ & $4(15)$ \\
\hline Mild pain & $4(15)$ & $3(11)$ & $6(23)$ & $9(35)$ & $7(27)$ & $3(11)$ & $16(62)$ & $9(35)$ & $13(50)$ & $17(65)$ \\
\hline Moderate pain & - & - & $1(4)$ & $1(4)$ & $8(31)$ & $5(19)$ & $4(15)$ & $11(42)$ & $9(35)$ & $3(12)$ \\
\hline Severe pain & - & - & - & - & $4(15)$ & $8(31)$ & $1(4)$ & $4(15)$ & $1(4)$ & $2(8)$ \\
\hline Very severe pain & - & - & - & - & - & $8(31)$ & - & - & - & - \\
\hline \multicolumn{11}{|l|}{ VS on movement $\dagger$} \\
\hline No pain & $21(81)$ & $21(81)$ & $12(46)$ & $11(42)$ & $2(8)$ & - & $1(4)$ & - & $1(4)$ & - \\
\hline Mild pain & $5(19)$ & $5(19)$ & $8(31)$ & $12(46)$ & $9(35)$ & $4(15)$ & $12(46)$ & $6(23)$ & $7(27)$ & $8(31)$ \\
\hline Moderate pain & - & - & $5(19)$ & $1(4)$ & $10(38)$ & $2(8)$ & $9(35)$ & $7(27)$ & $13(50)$ & $10(38)$ \\
\hline Severe pain & - & - & $1(4)$ & $2(8)$ & $3(11)$ & $11(42)$ & $4(15)$ & $9(35)$ & $5(19)$ & $8(31)$ \\
\hline Very severe pain & - & - & - & - & $2(8)$ & $9(35)$ & - & $4(15)$ & - & - \\
\hline \multicolumn{11}{|l|}{ Bromage scale } \\
\hline $1=\operatorname{nil}(0 \%)$ & - & - & - & - & $3(11)$ & $12(46)$ & $16(62)$ & $24(92)$ & $24(92)$ & $26(100)$ \\
\hline $2=$ partial $(33 \%)$ & - & - & - & - & $1(4)$ & $8(31)$ & $5(19)$ & $2(8)$ & $2(8)$ & - \\
\hline $3=$ almost complete $(66 \%)$ & $2(8)$ & $4(15)$ & $4(15)$ & $7(27)$ & $15(58)$ & $6(23)$ & $5(19)$ & - & - & - \\
\hline $4=$ complete $(100 \%)$ & $24(92)$ & $22(85)$ & $22(85)$ & $19(73)$ & 7 (27) & - & - & - & - & - \\
\hline
\end{tabular}

Data are reported as $\mathrm{n}(\%)$.

*Statistically significant differences were observed at $12(P=0.006)$ and $24 \mathrm{hrs}(P=0.04)$.

$\dagger$ Statistically significant differences were observed at $12(P=0.001)$ and $24 \mathrm{hrs}(P=0.05)$.

\$Statistically significant differences were observed at $12(P<0.001)$ and $24 \mathrm{hrs}(P=0.02)$. 


\section{RESULTS}

There were no significant differences between the CFNB and ON-Q groups according to age, sex, BMI, hemodynamic data, tourniquet, and surgery duration (Table 1). The consumption of propofol (in milligrams per kilogram per hour) and sufentanil (in micrograms per kilogram) did not differ significantly between the 2 groups (propofol CFNB: 4.2 [SD, 3.7], ONQ: 5.1 [SD, 4.3]; $P=0.4$; sufentanil CFNB: 0.09 [SD, 0.07], ON-Q: 0.08 [SD, 0.05 ]; $P=0.5$ ). No patient reported pain immediately after the surgery, and the leg block was complete in all patients.

The CFNB group reported significantly lower VAS pain scores than the ON-Q group: at rest at $12 \mathrm{hrs}$ and on movement at 12 and $24 \mathrm{hrs}$ after surgery (Table 2). Furthermore, the analgesia was significantly more effective in the CFNB group at 12 and 24 hrs after surgery when checked by using VS (Table 2). The movement of the operated leg was significantly more limited in the CFNB group at 12 and $24 \mathrm{hrs}$ after surgery as reported by the Bromage scale (Table 3). No significant differences were found in hemodynamic data during the surgery and during the postsurgery follow-up (data not shown). The mean number of PCRA ropivacaine boluses (CFNB group) was 4.1 [SD, 2.3]. The consumption of rescue doses of morphine and ketorolac was significantly lower in the CNFB group during the follow-up period (morphine: CFNB, 3.2 [SD, 2.1] boluses vs ON-Q, 6.4 [SD, 2.5] boluses; $P<0.001$; ketorolac: CFNB, 1.1 [SD, 1.0] boluses vs ON-Q, 2.5 [SD, 1.0] boluses; $P<0.001$ ). Nausea was the only postsurgery side effect observed, and there was no statistical difference between the groups (CFNB, 3 patients; ON$\mathrm{Q}, 4$ patients). There were no general or local adverse effects linked to the perineural (CFNB) or intra-articular injection $(\mathrm{ON}$ Q) of ropivacaine.

\section{DISCUSSION}

Our results show that CFNB provides superior analgesia as compared with the continuous intra-articular and wound infusion of local anesthetic using an ON-Q device. Furthermore, the overall rescue boluses of morphine and ketorolac were significantly higher in the ON-Q group. All patients found PCRA easy to use, and there were no complications observed. The pain scores were significantly higher at 12 and $24 \mathrm{hrs}$ after surgery in the ON-Q patients because of the resolution of the single-shot FNB as confirmed by the significant differences in the Bromage scores. The low level of analgesia achieved using ON-Q might be attributed to several factors: (1) the low dosage of the ropivacaine might be insufficient for the wide space covered by terminal nerve receptors (the articular capsule, the tibia, and femur bone holed for the positioning of the patellar tendon); (2) the in-aspiration drainage could have entrained also some of the ropivacaine; (3) the intra-articular distribution of the local anesthetic might not have been homogenous, resulting in uncovered areas; and (4) both lateral femoral and anteromedial tibial incision sites (used for the insertion of the grafted ligament) could not be anesthetized by the ropivacaine.

Because of the lack of the literature comparing CFNB and continuous intra-articular and wound infiltration of anesthetics after ACL reconstruction, it is impossible to compare our findings with those of other authors. In a systematic review, Moiniche et $\mathrm{al}^{7}$ evaluated the single intra-articular injection of local anesthetics compared with placebo or no treatment after arthroscopic knee surgery and reported weak evidence for the reduction of postoperative pain after intra-articular local anesthesia. ${ }^{7}$ Hoenecke et $\mathrm{al}^{4}$ performed continuous infiltration of the donor site of the patellar tendon using elastomeric pump at
$2 \mathrm{~mL} / \mathrm{hr}$ filled with $2.5 \mathrm{mg} / \mathrm{mL}$ bupivacaine and observed lower VAS pain scores at $12 \mathrm{hrs}$ as compared with our results from the ON-Q group and higher as compared with our CFNB group. Meanwhile, at $24 \mathrm{hrs}$, the VAS scores reported by these authors were similar to our findings on the ON-Q group. However, their patients received, in total, almost double the amount of intravenous opioids, as compared with our patients.

Alford and Fadale $^{3}$ reported less pain in patients with continuous intra-articular infusion of bupivacaine $2.5 \mathrm{mg} / \mathrm{mL}$ as compared with placebo or no intra-articular catheter placement after ACL reconstruction. In a nonrandomized and unblinded trial, Rasmussen et $\mathrm{al}^{8}$ studied continuous intra-articular injection of morphine and ropivacaine after total knee replacement. The intra-articular infusion led to a clinically relevant early improvement in motion and to a shortened hospital stay.

Several investigators have reported favorable results using FNB for postoperative pain management after open knee surgery and ACL repair. 6,9 In contrast to these positive results and to our findings, Schwarz et $a{ }^{5},{ }^{5}$ despite the statistically significant lower VAS pain scores observed performing the FNB, concluded that the difference was not clinically significant compared with intra-articular anesthetic injection after ACL repair. Both Tetzlaff et $\mathrm{al}^{10}$ and Williams et al, ${ }^{1}$ performing CFNB with bupivacaine (Tetzlaff et al: $0.65,1.25$, and 2.5 $\mathrm{mg} / \mathrm{mL}$ at $0.12 \mathrm{~mL} / \mathrm{kg} / \mathrm{hr}$ ) or levobupivacaine (Williams et al: $2.5 \mathrm{mg} / \mathrm{mL}$ at $5 \mathrm{~mL} / \mathrm{hr}$ ), after general (Tetzlaff et al) or spinal anesthesia (Williams et al), reported similar VAS scores in the postoperative period compared with our CFNB group at 12 hrs. Furthermore, Williams et $\mathrm{al}^{1}{ }^{1}$ reporting their experience (unpublished data, July 1999), observed that the VAS pain score was 1.8 while the block was effective, but the "rebound" VAS score was 5.3 immediately after nerve block resolution. These data are in accordance with our results at $12 \mathrm{hrs}$ when the nerve block was almost complete in the CFNB group and was resolving in the ON-Q group. Other authors concluded that CFNB both provides good analgesia (vs patient-controlled intravenous opioid analgesia) and leads to significantly better knee flexion, faster achievement of ambulation goals, and overall faster convalescence. ${ }^{11,12}$

Our study possesses some limitations. (1) No blinding: because of ethical and economical reasons, we preferred to avoid the placement of both CFNB and the ON-Q device. (2) Different doses of local anesthetics infused to perineural or intra-articular space: we preferred to select our dosages based on other author's reports of similar perineural ${ }^{2}$ and intra-articular ${ }^{3,8}$ anesthetic concentrations and infusion rates.

In conclusion, our results show that CFNB $(2 \mathrm{mg} / \mathrm{mL}$ of ropivacaine at $7 \mathrm{~mL} / \mathrm{hr}$ ) after ACL reconstruction provides a better level of analgesia compared with continuous intraarticular $(2 \mathrm{~mL} / \mathrm{hr})$ and patellar tendon wound $(2 \mathrm{~mL} / \mathrm{hr})$ infusions of $2 \mathrm{mg} / \mathrm{mL}$ of ropivacaine using an ON-Q device.

\section{ACKNOWLEDGMENTS}

The authors thank Dr. Gordon Filby and Mrs. Erjola Bakiri for editing the manuscript.

\section{REFERENCES}

1. Williams BA, Kentor ML, Vogt MT, et al. Reduction of verbal pain scores after anterior cruciate ligament reconstruction with 2-day continuous femoral nerve block: a randomized clinical trial. Anesthesiology. 2006;104:315-327.

2. Savoie FH, Field LD, Jenkins RN, Mallon WJ, Phelps RA 2nd. The pain control infusion pump for postoperative pain control in shoulder surgery. Arthroscopy. 2000;16:339-342. 
3. Alford JW, Fadale PD. Evaluation of postoperative bupivacaine infusion for pain management after anterior cruciate ligament reconstruction. Arthroscopy. 2003;19:855-861.

4. Hoenecke HR Jr, Pulido PA, Morris BA, Fronek J. The efficacy of continuous bupivacaine infiltration following anterior cruciate ligament reconstruction. Arthroscopy. 2002;18:854-858.

5. Schwarz SK, Franciosi LG, Ries CR, et al. Addition of femoral 3-in-1 blockade to intra-articular ropivacaine $0.2 \%$ does not reduce analgesic requirements following arthroscopic knee surgery. Can J Anaesth. 1999;46:741-747.

6. Iskandar H, Benard A, Ruel-Raymond J, Cochard G, Manaud B. Femoral block provides superior analgesia compared with intra-articular ropivacaine after anterior cruciate ligament reconstruction. Reg Anesth Pain Med. 2003;28:29-32.

7. Moiniche S, Mikkelsen S, Wetterslev J. A systematic review of intra-articular local anesthesia for postoperative pain relief after arthroscopic knee surgery. Reg Anesth Pain Med. 1999;24:430-437.

8. Rasmussen S, Kramhoft M, Sperling K, Pedersen J. Increased flexion and reduced hospital stay with continuous intraarticular morphine and ropivacaine after primary total knee replacement. Acta Orthop Scand. 2004;75:606-609.

9. Edkin BS, McCarty EC. Analgesia with femoral nerve block for anterior cruciate ligament reconstruction. Clin Orthop. 1999;369:289-295.

10. Tetzlaff JE, Andrish J, O’Hara J Jr, Dilger J, Yoon HJ. Effectiveness of bupivacaine administered via femoral nerve catheter for pain control after cruciate ligament repair. J Clin Anesth. 1997;9: 542-545.

11. Singelyn FJ, Deyaert M, Pendeville E, Gouverneur JM. Effects of intravenous patient-controlled analgesia with morphine, continuous epidural analgesia, and continuous three-in-one block on postoperative pain and knee rehabilitation after unilateral total knee arthroplasty. Anesth Analg. 1998;87:88-92.

12. Capdevila X, Barthelet Y, Biboulet P, Ryckwaert Y, Rubenovitch J, d'Athis F. Effects of perioperative analgesic technique on the surgical outcome and duration of rehabilitation after major knee surgery. Anesthesiology. 1999;91:8-15. 